\title{
La question de la neutralité portugaise 1939-1945
}

\section{János Sáringer}

Dans mon intervention j'esquiserrai les points de jonction qui peuvent servir comme explication á la neutralité portugaise. Je voudrais simplement formuler ici les hypothèses qui sont énoncées plus en détail dans ma thèse de doctorat.

Pourquoi dit-on que le Portugal était neutre durant la Seconde Guerre mondiale? La neutralité portugaise put une situation de facto bien qu'elle n'ait été jamais déclarée. En septembre 1939, au début de la Seconde Guerre mondiale «les portes du vieux continent se sont fermées». La guerre a commancé d'abord en Europe, pous s'entendre ensuite au monde entier. Qurant la guerre, en Europe, il n'y a eu que cinq pays qui sont restés neutres: la Turquie, la Suisse, la Suède, l'Espagne et le Portugal. Dès l'éclatement de la guerre, le Portugal a montré une neutralité bienveillante vis-à-vis de la Grande-Bretagne et, n'a pas adopté de positor négative par rapport á l'Allemagne. Cette attitude a changé dès 1941 et cette neutralité bienveillante s'est tournée plutôt vers l'Allemagne. A partir du printemps 1943 la direction de la politique étrangère portugaise prendre une nouvelle tournure et on observe un rapprochement vers les Alliés. Quelle peut en être l'explication?

On peut discerner trois principales directions de neutralité qui conditionne, en fait, ce statut de neutralite: premièrement garantir et observer les obligations contractées envers les deux camps ennemis, deuxièmement éviter quoi qu'il arrive une agression étrangère, troisièmement conduire une stratégie qui puisse assurer le maintien de la neutralité du territorie.

Il faut savoir que neutralité du Portugal à cette époque-là n'était pas évidente, le gouvernement de Lisbonne était pragmatique, la neutralité ne voulait pas dire une situation constante. Jusqu'en mai 1943, la neutralité s'est tournée plutôt vers l'Allemagne, même si plus tard Lisbonne s'est rapprochée des Alliés. Cela est prouvé par le fout que Salazar et son entourage ont interprété la neutralité en faveur de la partie dont la victoire était probable. On peut dire que le pragmatisme de Lisbonne était fluctuant, sa politique a changé comme es marées: elle a directement suivi directement l'alternance des succès et des échecs militaires et diplomatiques des Alliés et de l'Axe. A tout cela il faut ajouter l'obligation économique qui a aussi contribué dans une longe mesure.

On peut aussi trouver des antécédents de la politique de neutralité du gouvernement portugais dans la guerre civile espagnole. En matière d'intérêts politiques, le Portugal conservatif et la GrandeBretagne libérale se sont retrouvés aux antipodes l'un de l'autre. Tandis que le Portugal a maintenu Franco, la Grande-Bretagne a maintenu les forces de la république. A propos du Portugal et de la Grande-Bretagne, on peut caractériser l'époque comprise entre 1936 et 1939 comme une periode oú les gouvernements ont fait de la politique d'《intervention» ou de «non-intervention». Le gouvernements portugais s'est trouvé devant une décision difficile á prendre, parce que la politique d'«intervention» aurait pu désunir les relations alliées longues de 5 siècles entre Lisbonne et Londres. Les intérêts commerciaux et économiques du Portugal conraignaient aussi ce dernier á mener une politique à côté de Londres et non pas en face des intérêts britanniques car la marine britannique, les bases maritimes et océaniques de l'Angleterre assuraient les transports commerciaux portugais. La marine britannique jouait aussi un grand rôle dans la continuité des contacts économiques entre la métropole portugaise et les colonies extrême-orientales et africaines.

La présence allemande dans la péninsule est concrèt depuis les années 30 . Le rôle de l'aide militaire allemande à Franco pendant la guerre civile espagnole est connu. Et le matériel de guerre a afflué des ports portugais à l'intention des révoltants. Salazar a envoyé à l'intention de Franco une légion de volontaires bien que les deux pays aient eu des orientations politiques internationales différentes. Afin de régler les opinions différentes, les chefs des deux pays ont signé un pacte ibérique en mars 1939 qui contenait un pacte d'amitié et de non-agression. ${ }^{1}$

\footnotetext{
1 Catarina de ALMADA SADANHA SANTOS BARREIROS CARDOSO: A estratégia do Estado Novo. Lisboa, 1996, (Dissertação de Mestrado em Estratégia)
} 
La présence allemande au Portugal est peu connue dons l'historiographie hongroise. Les positions allemandes au Portugal sont palpables dès le milieu des années 1930. Elles était économiques, culturelles, mêmes politiques. Les relations des deux pays ont été renforcées par la présence au Portugal de l'Académie lusitano-allemande (à Coimbra, à Porto, au Cap-Verte et aux Açors), l'Institut culturel allemand (à Lisbonne, à Porto), le Club allemand (à Lisbonne), la Croix rouge allemande et l'Hopital allemand (à Lisbonne), le Collège de l'Allemagne (à Lisbonne et à Porto) et le Hitlerjugend. Meus voudrions ici mettre sui il fait que l'Académie lusitano-allemande était de facto la base du Portugal de la NSDAP ainsi que de la Gestapo. ${ }^{2}$

La relation commerciale des deux pays a été coordonnée par l'Usine Göring (avant tout pour le transport du volfram), par la Société Minière du Portugal du Nord et aussi par plusieurs groupes capitalistes industrliels allemands qui étaient présents dans le pays (par exemple le Kisher, le Wimer, le Meyer, le Hubman, le Zickerman). En outre, des groupes d'intéressés allemands possédaient des territoires agricoles immenses. L'industrie de guerre allemande a aussi importé plusieurs produits dons le pays, par exemple des armes Mauser, des systèmes optiques Zeiss, des avions de type Junkers.

Lors de l'éclatement de la guerre du point de vue britannique, les réactions portugaises éont été encourageantes. Salazar déclare le 1er septembre 1939 qu'ils est prêta aider de toutes les manières possibles es alliés britanniques, Il glorifie aussi «le sacrifice héroïque de la Pologne». En octobre Salazar met au jour le message de Berlin dont le fond cetait que l'Allemagne cetait prête à garantir l'integrité territoriale du Portugal et de ses colonies d'outre-mer si le Portugal restait neutre. ${ }^{3}$

A cette épque-là Londres est passé encore sur que le Portugal donnait toutes les services à l'Allemagne qui était permis, mais non pas obligatoires pour les pays neutres. On peut expliquer cela par le fait que la Grande-Bretagne n'avait pas encore besoin de l'aide du Portugal pour mointenir de sa position atlantique. La principale maison de Londres était d' 'exploiter le plus possible la bienveillance des Portugais»».

A la fin de 1939, la neutralité du Portugal fut aussi trés favorable pour le gouvernement espagnol. La désicion de Lisbonne d'entre en guerre aux côtés des Alliés n'aurait pas dérangé la position de «non-belligérant» de Madrid.

Dans les années 1930 l'économie portugaise a connu un déficit grave. Le régime de Salazar du point de vue économique était assez boiteux ce qui s'explique par la particularité du régime. Depuis 1929 , le système «clearing» introduit dans commerce international avait rendu plus difficile le commerce portugais parce que l'économie portugaise souffrait d'un manque en or. La convertibilité de la monnaie portugaise était beaucoup plus faible que celle des monnaies américaine, anglaise, française ou alemande. L'éclatement de la guerre et la prospérité économique qui a suivi ont permis au Portugal de jeunis d'une occasion bénéfique pour lui-même la logique de la guerre avait une poussée des circonstances et il y avait la possibilité de reconvertir l'économie et le commerce portugais en production et transport de guerre.

D'après des données statistiques portugaises on peut constater que tandis qu'entre 1931 et 1941 le taux d'exportation et d'importation était négatif, entre 1941 et 1943 il était positif. Ensuit le bilan des exportations et des importations est redevenu négatif. En continuant l'observation, on peut constater que le bilan positif entre 1941 et 1943 a resulté du commerce qui avait été fait avec l'Allemagne et l'Angleterre. ${ }^{4}$

Le blocus de l'Angleterre, et la chute de la France ont contraint les Allies á prendre des mesures économiques (à côté des mesures militaires). Du point de vue de l'économie portugaise, la soi-disante «liste noir» publiée par les Alliés en 1940 est un événement fait date dans l'histoire.Cette liste contenait des produits importants d'un paint de une stratégique tout qui dont interdit de transporter aux poussonces de l'Axe, et avant tout du volfram, des conserves de poisson ou du tissu. L'autre événement important, fut l'introduction du soi-disant système navicerts, selon lequel tous les bateaux approchant l'Europe devaient avoir une attestation de transport maritime ce qui a permis de obressu une liste exhaustive des navicerts. Le but du système navicerts était - entre autres - d'empécher la réexportation des productions portugaises et des autres pays aux puissaneur de l'Axe.

\footnotetext{
${ }^{2}$ António José TELO: A neutralidade portuguesa e o ouro nazi. Lisboa, Quetzal Editores, 2000

${ }^{3}$ Joaquim VERISSIMO SERRÃO: Historia de Portugal (1935-1941). Lisboa, Editorial Verbo, XIV. vol 385-453 p.

António José TELO: A neutralidade...85-219 p.
} 
La relation commerciale entre l'Allemagne et le Portugal s'est équilibrée entre 1939 et 1941. Á cette époque-là, Berlin ne s'intéressait pas trop ni aux produits portugais, ni au volfram parce qu'il les achetait á l'Union soviétique et au Japon. Après 1941 les points de vue allemand et portugais se sont rapprochés. Ce rapprochement la signature d'un contrat comercial portugo-allemand en Janvier 1942. Celui-ci s'appliquait surtout au transport du volfram. Le gouvernement portugais demandait é êlle payé en retour en franc suisse et en or à via des banques suisses et le Saint-Siège. Ce-ci explique pourquoi le bilan de l'économie portugaise était positif entre 1941 et 1943. On ne peut pas omettre mentionner bien sûr le volume du commerce anglo-portugais qui a contribué dans une long mesure à faire que l'indice économique portugais était positif. L'afflux á or allemand a stabilisé l'économie portugaise.

Le primtemps de 1943 est un tournant dans la guerre quand les positions atlantiques et africaines des Alliés se sont renforcées militairement. 1'Est l'Armée rouge a contre-attaqué et a refoulé graduellement les troupes de la Wermacht. Les Alliés ont alors du exercer des pressions diplomatiques sur leurs alliés ou sur les pays neutres. Les dirigeants de la politique étrangère accredités à Lisbonne. Au du printemps de 1943, la Grande-Bretagne a demandé au gouvernement portugais d'autoriser à l'implantation d'une base aux Açors. Le cabinet de Salazar s'est trouvé devant un dilemme car avec la construction de la base il deitait abandonner sa position neutre et à cause de la fin possible du commerce fait avec l'Allemagne il pouvait perdre une recette sérieuse. La situation était encore plus compliquée à cause du projet du Foreign Office qui prévoyant l'invasion des îles en cas refuse de Salazar. On ne peut pas connaitre exactitude la nature des informations du Ministère des Affaires étrangères portugais à propos du projet d'invasion mais il est certain que le gouvernement portugais a recherché un «modus vivendi». On pense que dans la décision finale du gouvernement portugais l'événement diplomatique qui se déroule en septembre 1943 a joué un rôle important. Notmment, l'Italie et les Allies ont signé un pacte d'armistice á la suite duquel Hitler a perdu son bastion en Italie du Sud. Enfin, en octobre 1943, Salazar et son cabinet ont abandonné les Açors aux Alliés, mais il a continué les transports avec l'Allemagne. En dépit de la crise économique de 1943 l'armement de l'armée portugaise en matériel de guerre anglais s'est poussuivi a continué à un rythme accéléré. Par conséquent à la fin de 1943, on ne peut pas considérer le Portugal comme neutre de facto bien que l'ambition forte de la politique officielle ait été d'éloigner le pays de la guerre européenne. La fin de 1943 a été un tournont dans la politique étrangère (économique) portugaise. La Grande Bretagne exercait une faite pression sur le Portugal. Dès le début de 1944 le Portugal a baissé graduellement ses acheminements en marchandise à l'Allemagne et parallèlement, il renforcé la ligne anglo-saxonne dans sa politique étrangère.

Engrise de conclusion á la question qui a été possée au début de notre intervention, on peut répondre que le gouvernement portugais a metté une politique pragmatique. Juridiquement le statut de neutralité du Portugal n'était pas non plus formel, car sa situation peut-on périodiser. Nous estemons pense que la réponse est complexe: l'un des facteurs décisifs est la bonne entente séculaire sur base d'intérêts mutuels entre le Portugal et la Grande-Bretagne, l'autre facteur est la contrainte de stabilisation de l'économie portugaise, et le troisième facteur est la possibilité du règlement d'après guerre. Ce sont ces facteurs qui ont conditionné les directions de la politique étrangère portugaise durant la Seconde Guerre mondiale. 